\title{
Patient Expectations of Visiting Department of Oral Medicine for Burning Mouth Syndrome: Relationship between Expectations and Clinical and Psychological Characteristics
}

\author{
Hye-Kyoung Kim, Mee-Eun Kim
}

Department of Oral Medicine, College of Dentistry, Dankook University, Cheonan, Korea

Received November 22, 2019

Revised November 29, 2019

Accepted December 3, 2019

\section{Correspondence to:}

Mee-Eun Kim

Department of Oral Medicine, College of

Dentistry, Dankook University, 119 Dandae-

ro, Dongnam-gu, Cheonan 31116, Korea

Tel: +82-41-550-1915

Fax: +82-505-434-7951

E-mail:meunkim@dankook.ac.kr

https://orcid.org/0000-0001-9332-532X
Purpose: The aims of this study were to investigate the expectation of patients with burning mouth syndrome (BMS) and assess the relationship between patient expectations and clinical and psychological characteristics.

Methods: Eligibility was retrospectively assessed on 93 patients with BMS. A total of five questionnaires on patients' expectation for a visit, pain, sleep quality (Pittsburgh Sleep Quality Index), cognition (Pain Catastrophizing Scale) and psychological distress (Symptom Checklist-90 revised) were measured.

Results: Sixty-five patients were included in this study. The top 3 priorities of expectation for a treatment visit to the Department of Oral Medicine were as following; "I want my pain to be free"; "I want to understand why it hurts"; "I want to communicate better with doctors about pain". Patients with priority of pain relief showed poor sleep quality than patients who are more interested in the disease related information.

Conclusions: To improve treatment outcomes of BMS, clinicians should improve their own understanding of patients who are suffering from BMS. Inquiring about the patient expectations may be one of them, and it would contribute to the enhancement of patients' overall well-being.

Key Words: Burning mouth syndrome; Expectation; Surveys and questionnaires

\section{INTRODUCTION}

Burning mouth syndrome (BMS), also called glossodynia or stomatodynia, is one of the most common, but debilitating oral condition that is characterized by constant burning sensations in the oral mucosa, which does not appear to be abnormal by visual examination or laboratory tests [1,2]. In addition to burning pain, it simultaneously presents a variety of symptoms including xerostomia, dysgeusia, and even psychological distress. However, compared to the great disability of patients, the lack of consensus on the etiology of BMS has made it extremely difficult to establish a correct treatment protocol. Many previous studies have reported that poorly treated BMS can cause patients to visit several hospitals to seek further medical treatment and therefore, has a significant negative impact on the quality of individual life [3-5]. As mentioned above, patients with BMS may have a long history of oral pain and experience repetitive treatment failure.

Considering the natural course that a patient feels anxiety about a refractory pain of unknown etiology, it is not surprising that BMS, as a chronic pain, is frequently involved with stressful life events and psychological conditions including depression, anxiety, neuroticism and cancerphobia $[2,6]$. These intractable clinical problems that cannot be fully explained by biological processes are also a challenge for

Copyright (c) 2019 Korean Academy of Orofacial Pain and Oral Medicine. All rights reserved.

(c) This is an open-access article distributed under the terms of the Creative Commons Attribution Non-Commercial License (http://creativecommons.org/licenses/by-nc/4.0/), which permits unrestricted non-commercial use, distribution, and reproduction in any medium, provided the original work is properly cited. 
clinicians and thus, even little information on the patients might be a great help to clinicians.

In this context, assessing and understanding the expectations and priorities that BMS patients have may help clinicians to optimize treatment and support patients and finally improve patient-reported outcomes. However, to the authors's knowledge, little is known about the expectations of patients with BMS.

Therefore, the aims of this study were to investigate the expectation of patients with BMS and assess the relationship between patient expectations and clinical and psychological characteristics.

\section{MATERIALS AND METHODS}

\section{Subjects}

This study was the second part of a study that investigated characteristics of patients with BMS. Part I of the study also examined the pain intensity and interference, sleep quality and psychological distress with the same patients, and it has been previously published [7]. Details of subjects

Table 1. Demographics and pain characteristics

\begin{tabular}{lc}
\hline \multicolumn{1}{c}{ Variable } & Value $(n=65)$ \\
\hline Age $(y)$ & $54.8 \pm 14.8$ \\
Female & $37(56.9)$ \\
Pain duration (mo) & $21.8 \pm 30.7$ \\
BPI & $4.2 \pm 2.6$ \\
Pain severity & $4.4 \pm 3.1$
\end{tabular}

BPI, Brief Pain Inventory.

Values are presented as mean \pm standard deviation or number (\%). and procedures are described in Lee's study [7].

Between January 2017 to August 2018, a total of 93 patients were diagnosed as BMS at the Department of Oral Medicine of Dankook University Dental Hospital according to the guidelines from the International Classification of Headache Disorders, 3rd edition (beta version) [8]. Inclusion and exclusion criteria are well described in Lee's study [7]. To rule out the possible secondary causes of BMS, blood test, candida test and salivary flow rate test were performed.

All patients were asked to complete a number of questionnaires prior to initial examination as part of standard protocol of the Department of Oral Medicine of Dankook University Dental Hospital in the waiting room.

Informed consent for using their clinical data for the research purpose prior to the initial examination was obtained. Data were collected retrospectively from clinical records and questionnaires of the eligible patients. The current study was approved by the Institutional Review Board committee of Dankook University Dental Hospital (IRB no. DKUDH IRB 2018-09-001).

\section{Assessment of Patients' Expectation}

Patients' expectation of visiting to the Department of Oral Medicine was assessed using the self-reported questionnaire (Table 1). This questionnaire includes a total of fourteen questions associated with expectation. Patients can select a total of six questions out of the 14 items in the order of their priority. Then, the sum ratio of top three priorities for each question was calculated and used for further comparison.

Table 2. The questionnaire and scale of patient's expectation of the first visit to the Department of Oral Medicine

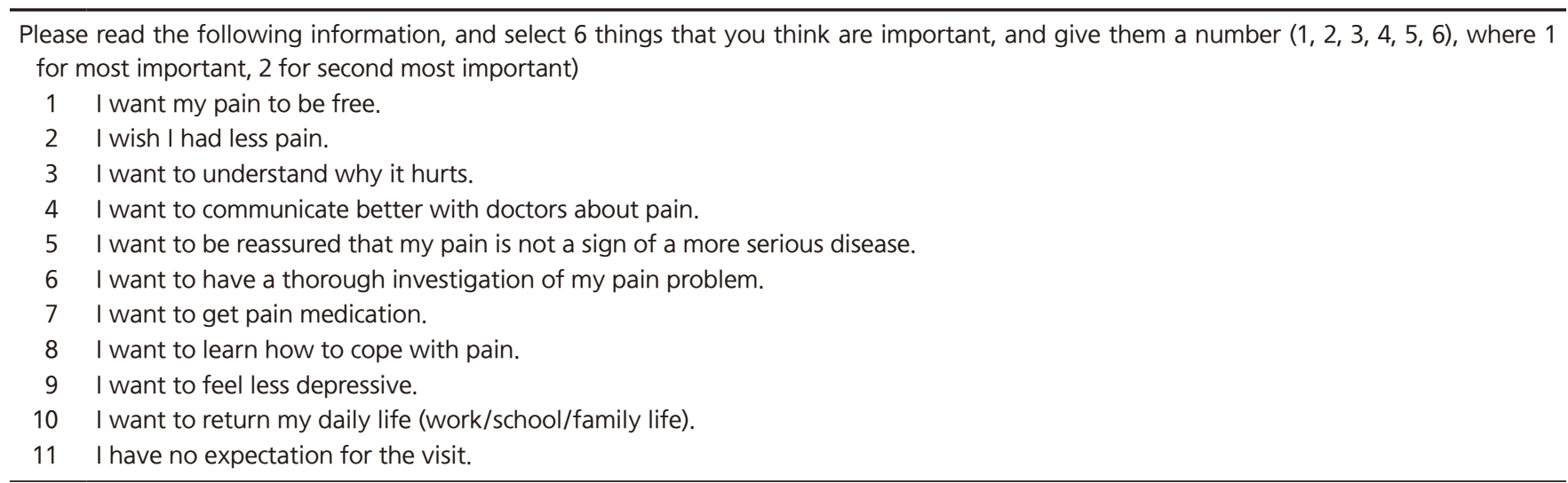




\section{Assessment of Pain and Mental Condition}

The four questionnaires on pain and mental condition used in this study are the same as those used in part I [7], and are described in detail elsewhere. The Brief Pain Inventory (BPI) is a valid tool for assessment of pain [9]. In this study, Korean version of BPI [10] was used to measure the pain intensity and pain interference of patients. The Pittsburg Sleep Quality Index (PSQI), a valid and reliable questionnaire for evaluation of subjective sleep quality, was used to measure the sleep quality of patients with BMS in this study [11]. Catastrophizing thinking was measured by pain catastrophizing scale (PCS) [12]. It consists of 3 components including rumination, magnification and helplessness and one total score. Psychological conditions including depression and anxiety were evaluated using the Symptom Checklist-90 revised (SCL-90R) [13]. It measures nine subtypes of psychopathology.

\section{Data Analysis}

Considering that the possible influence of sex, age and pain duration on the pain experience, data from age, sex, pain duration to pain severity and interference, PCS, PSQI, and SCL-90R from questionnaires were used for statistical analyses. Demographics and pain characteristics of the patients were presented as percentage and frequency. The percentage of expectation rank for each question in the questionnaire were calculated using descriptive statistics. Comparison between groups was performed using the Independent sample t-test. Statistical analyses were

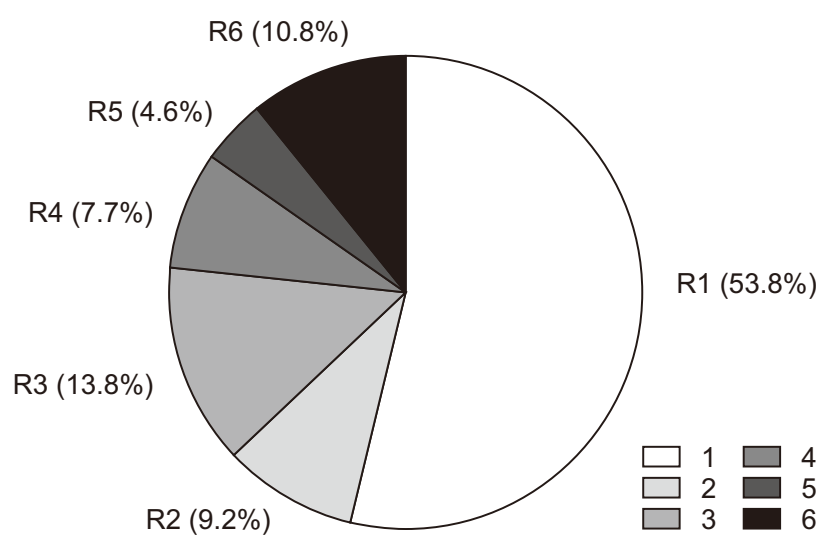

Fig. 1. Proportion of expectation rank for the question "I want my pain to be free". $R$ indicates rank of the patient's expectations for the question. $\%$ of rank $1+2+3=76.8 \%$ conducted using IBM SPSS Statistics for Windows, Version 21.0 (IBM Co., Armonk, NY, USA) and statistical significance was set at $\mathrm{p}=0.05$.

\section{RESULTS}

\section{Patient Characteristics}

Demographics of the study population are described in Table 2. Ninety-three eligible patients were enrolled in this study. Among them, 65 patients were finally included according to the inclusion and exclusion criteria. 56.9\% of the patients were female. The mean \pm SD age and disease duration were $54.8 \pm 14.8$ years and $21.8 \pm 30.7$ months, respectively. At baseline, the mean \pm SD pain severity and pain interference measured by BPI were $4.2 \pm 2.6$ and $4.4 \pm 3.1$, respectively.

\section{Patient Expectation Ratings}

Patients rated priorities on their expectations of visiting Department of Oral Medicine. The Fig. 1-7 show the proportion of patients' expected ranking from the first to sixth and the sum ratio of the first, second and third rank for each question in the expectation questionnaire.

In the question "I want my pain to be free", the sum percentage of expectation rank of 1, 2, and 3 was 76.8\% (Fig. 1). For the question "I wish I had less pain", 29.2\% was recorded for the sum percentage of expectation rank of 1,2 , and 3 (Fig. 2). In Fig. 3, the sum percentage of expectation rank of 1, 2, and 3 for the question "I want to understand

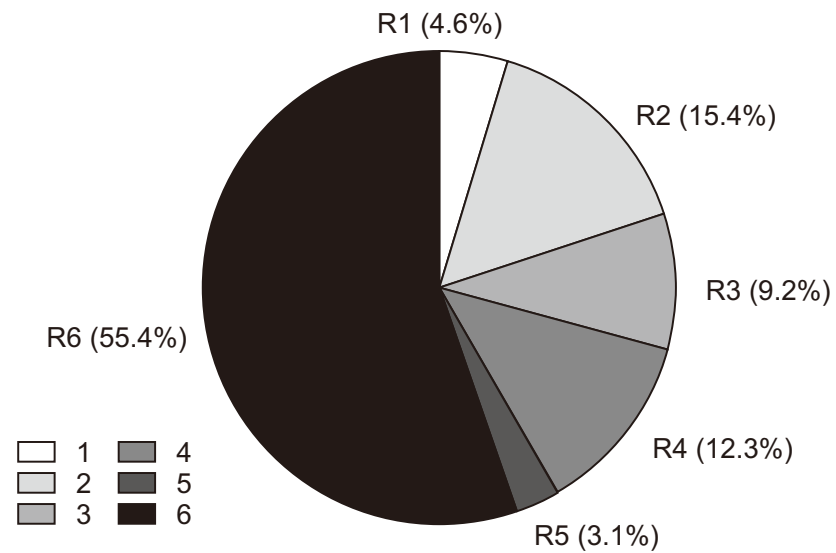

Fig. 2. Proportion of expectation rank for the question "I wish I had less pain". $\mathrm{R}$ indicates rank of the patient's expectations for the question. $\%$ of rank $1+2+3=29.2 \%$ 


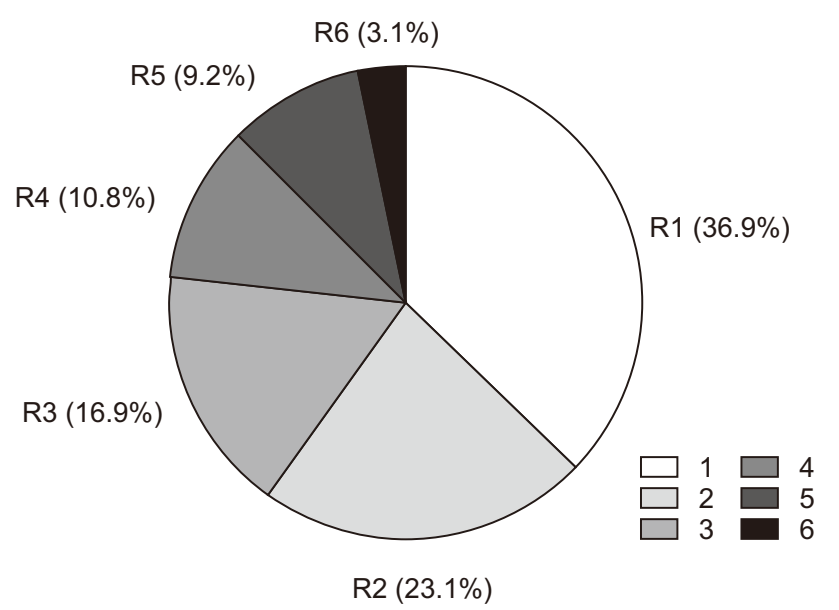

Fig. 3. Proportion of expectation rank for the question "I want to understand why it hurts". $R$ indicates rank of the patient's expectations for the question. $\%$ of rank $1+2+3=76.9 \%$

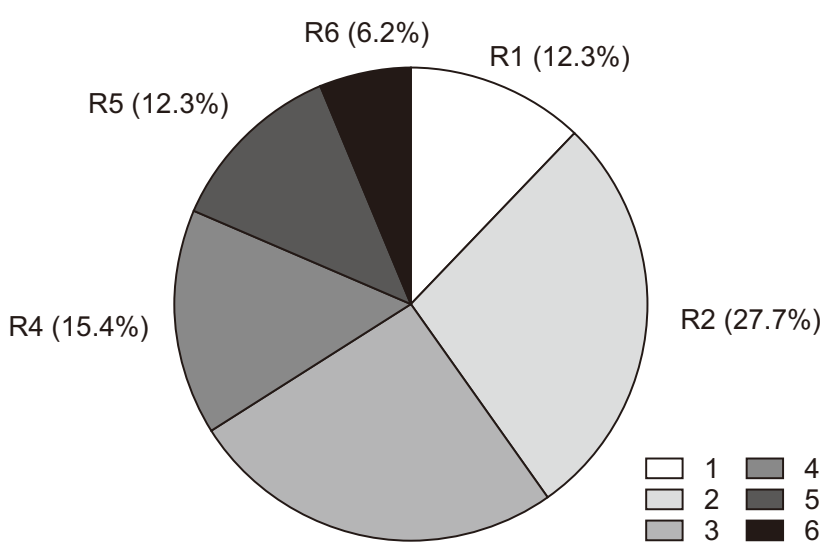

R3 (26.2\%)

Fig. 4. Proportion of expectation rank for the question "I want to communicate better with doctors about pain". R indicates rank of the patient's expectations for the question. $\%$ of rank $1+2+3=66.2 \%$

why it hurts" was 76.9\%, which showed the highest proportion of rank 1,2 and 3 with question "I want my pain to be free" (76.8\%). The question "I want to communicate better with doctors about pain" showed $66.2 \%$ of expectation rank of 1, 2, and 3 (Fig. 4). Proportion of expectation rank for the question "I want to be reassured that my pain is not a sign of a more serious disease", "I want to have a thorough investigation of my problem" and "I want to get pain medication” were 21.6\% (Fig. 5), 18.4\% (Fig. 6) and 10.7\% (Fig. 7).

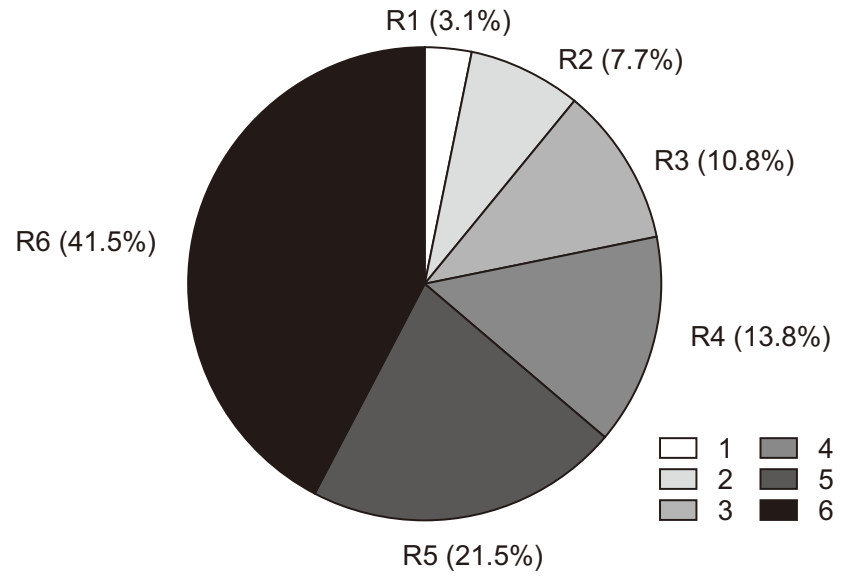

Fig. 5. Proportion of expectation rank for the question "I want to be reassured that my pain is not a sign of a more serious disease". $\mathrm{R}$ indicates rank of the patient's expectations for the question. \% of rank $1+2+3=21.6 \%$

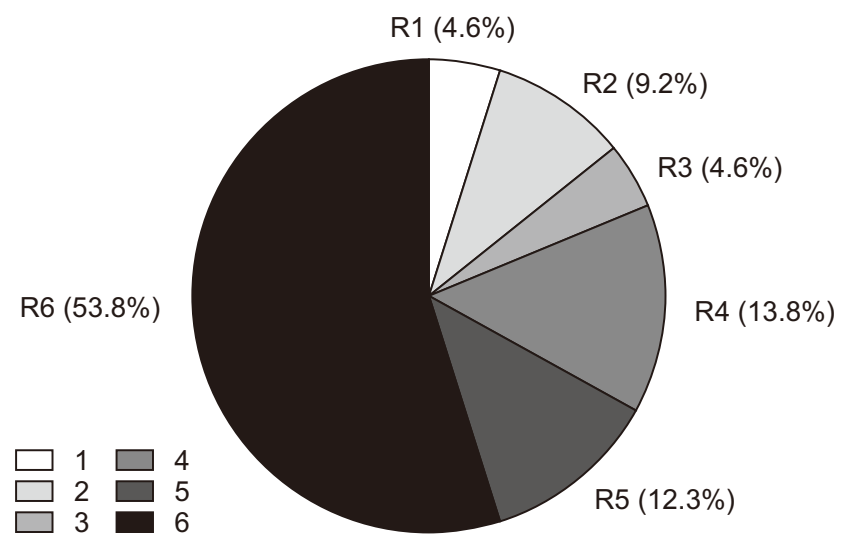

Fig. 6. Proportion of expectation rank for the question "I want to have a thorough investigation of my pain problem". R indicates rank of the patient's expectations for the question. \% of rank $1+2+3=18.4 \%$

\section{Comparison of Clinical and Psychological \\ Characteristics between Two BMS Groups with Different Expectation}

Table 3 shows the clinical and psychological characteristics differences between two groups with different patient's expectation.

Of the four expectations that patients value most, patients with the highest expectations in question 1 (I want my pain to be free) and 2 (I wish I had less pain) were stratified into group $1(n=33)$. On the other hand, patients who have the highest expectations in question 3 (I want to understand why it hurts) and 4 (I want to communicate better with doctors about pain) were classified into group $2(n=32)$. In this 


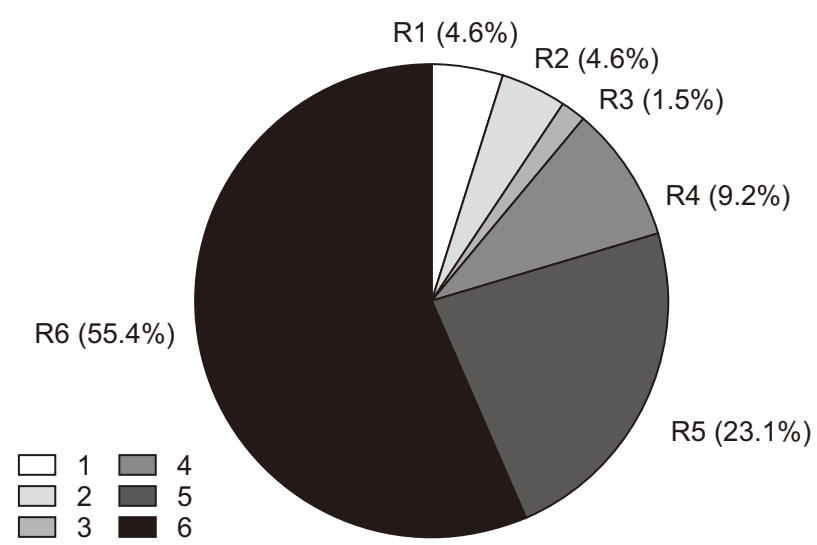

Fig. 7. Proportion of expectation rank for the question "I want to get pain medication". $\mathrm{R}$ indicates rank of the patient's expectations for the question. $\%$ of rank $1+2+3=10.7 \%$

stratification, group 1 indicates the patients who focused on being free of pain, understanding the cause of pain, as an expectation, was main characteristics of group 2.

Two groups did not show statistical differences between clinical characteristics including sex $(\mathrm{p}=0.132)$, age ( $p=0.461)$, duration ( $p=0.183)$, pain severity $(\mathrm{p}=0.122)$ and pain interference ( $p=0.446)$. Pain catastrophizing scores including three dimensions of magnification $(\mathrm{p}=0.946)$, rumination $(\mathrm{p}=0.590)$ and helplessness $(\mathrm{p}=0.866)$ also did not differ between two groups. Multiple dimensions of SCL90R were compared between two groups. Although the mean Somatization score of group 1 was higher than that of group 2, it did not reach statistical significance ( $\mathrm{p}=0.061)$. Like somatization, other dimensions of SCL-90R did not statistically differ between two groups. On the other hand, the mean score of global PSQI index was significantly higher in group 1 than group $2(\mathrm{p}=0.035)$.

\section{DISCUSSION}

Previous studies revealed that BMS has a negative impact on the quality of life of patients, but individualized management can improve the patient-reported outcomes including intraoral pain and quality of life [5,14]. For tailored approach, knowledge on the priorities and expectations of patients is valuable.

In this study, the top 3 priorities of expectation for a treatment visit to the Department of Oral Medicine were as
Table 3. Comparison of clinical and psychological characteristics between two groups with different patient's expectation $(n=65)$

\begin{tabular}{|c|c|c|c|}
\hline Variable & $\begin{array}{c}\text { Group } 1 \\
(n=33)\end{array}$ & $\begin{array}{c}\text { Group } 2 \\
(n=32)\end{array}$ & $p$-value \\
\hline \multicolumn{4}{|l|}{ Clinical characteristics } \\
\hline Sex & & & 0.132 \\
\hline Age & $56.4 \pm 14.6$ & $53.5 \pm 15.7$ & 0.461 \\
\hline Duration & $16.9 \pm 25.3$ & $27.8 \pm 36.4$ & 0.183 \\
\hline Pain severity & $3.7 \pm 2.4$ & $4.7 \pm 2.7$ & 0.122 \\
\hline Pain interference & $4.1 \pm 2.8$ & $4.7 \pm 3.4$ & 0.446 \\
\hline \multicolumn{4}{|l|}{ Pain catastrophizing } \\
\hline PCS_magnification & $5.4 \pm 3.5$ & $5.5 \pm 3.6$ & 0.946 \\
\hline PCS_rumination & $7.8 \pm 5.2$ & $7.1 \pm 5.5$ & 0.590 \\
\hline PCS_helplessness & $9.8 \pm 7.4$ & $10.1 \pm 7.3$ & 0.866 \\
\hline \multicolumn{4}{|l|}{ Sleep quality } \\
\hline PSQI & $8.6 \pm 4.6$ & $6.4 \pm 3.3$ & 0.035 \\
\hline \multicolumn{4}{|l|}{ SCL-90R } \\
\hline Somatization & $50.8 \pm 14.3$ & $45.4 \pm 6.9$ & 0.061 \\
\hline depression & $45.5 \pm 12.6$ & $44.5 \pm 10.0$ & 0.733 \\
\hline Anxiety & $48.4 \pm 14.0$ & $44.2 \pm 7.4$ & 0.135 \\
\hline Obsessive-compulsive & $46.2 \pm 14.5$ & $14.3 \pm 9.1$ & 0.539 \\
\hline Interpersonal sensitivity & $43.6 \pm 13.4$ & $43.8 \pm 8.3$ & 0.936 \\
\hline Hostility & $47.2 \pm 13.5$ & $43.2 \pm 3.3$ & 0.103 \\
\hline Phobic-anxiety & $50.0 \pm 18.7$ & $45.6 \pm 7.5$ & 0.219 \\
\hline Paranoid & $45.1 \pm 13.8$ & $42.2 \pm 6.2$ & 0.277 \\
\hline Psychosis & $46.9 \pm 13.6$ & $44.6 \pm 6.8$ & 0.407 \\
\hline
\end{tabular}

PCS, pain catastrophizing scale; PSQI, Pittsburg Sleep Quality Index; SCL-90R, Symptom Checklist-90 revised.

Values are presented as mean \pm standard deviation.

Group 1 indicates "I want my pain to be free" (CC1), "I wish I had less pain" (CC2). Group 2 indicates "I want to understand why it hurts" (CC3), "I want to communicate better with doctors about pain (CC4).

$\mathrm{p}$-values were obtained from the Independent sample t-test.

following; "I want my pain to be free"; "I want to understand why it hurts"; "I want to communicate better with doctors about pain".

Among 65 patients, no one patients first visited the Department of Oral Medicine due to oral pain. Despite previous conservative treatment, patients had high expectations about relieving pain and understanding the cause of pain. Patients prioritized for the complete disappearance of pain $(76.8 \%)$ rather than less pain (29.2\%). Patients also expected it was important to know why I am sick (76.9\%) and to listen to the doctor's detailed explanation (66.2\%) rather than knowing that my illness was not serious $(21.6 \%)$ or get a prescribed medicine (10.7\%). Generally, it is reported that BMS patients show a great fear of oral cancer called cancerphobia, and therefore, treatment should be focused on supportive reassurance that it is not a serious condition 
such as oral cancer [15]. Interestingly, this study presents that BMS patients do not give priority to this point.

In this study, two groups with different priority of painfocused and information-focused expectation were stratified and compared of clinical and psychological characteristics at baseline. Two groups did not show any differences in clinical aspects including demographic and pain characteristics. Also, there was no differences in pain catastrophizing and psychological distress such as depression and anxiety between two groups. Interestingly, the mean score of global PSQI index was significantly higher in pain-focused group than information-focused group ( $p=0.035)$. Adamo et al. [16] reported the high prevalence of poor sleep quality in BMS patients. Furthermore, strong positive relationship between poor sleep quality and pain experience in chronic orofacial pain was demonstrated in previous studies [16-19]. Considering this bidirectional association between sleep and pain, sleep problem should be more considered in BMS patients with the expectation focused on pain.

Overall, patients had very high expectations for relieving pain and information. Despite similar pain scores and psychological characteristics, patients focused on pain demonstrated poor sleep quality than patients with informationoriented expectations. These results may have implications for prioritizing treatment protocol. For patients with painoriented expectations, active pain-relieving approach with evaluation of comorbid sleep problem should be focused first. On the other hand, providing adequate medical information including disease characteristics and medication effects and related education on pain control and improving functionality in daily activities should be focused for patients who prioritize disease-related information. This education and reassurance may help the patients follow the treatment plan and medication adherence.

The question of whether treatment based on patient's expectations improves outcomes deserves further research. In addition, further studies are required to investigate whether treatment prognosis differ between patients with different expectations despite the same pain levels and emotional stress, and whether treatments considering expectation priorities are effective.

In conclusion, patients with priority of pain relief showed poor sleep quality than patients who are more interested in the disease related information. To improve treatment outcomes of BMS, clinicians should improve their own understanding of patients who are suffering from BMS. Inquiring about the patient expectations may be one of them, and it would contribute to the enhancement of patients' overall well-being.

\section{CONFLICT OF INTEREST}

No potential conflict of interest relevant to this article was reported.

\section{ORCID}

\author{
Hye-Kyoung Kim \\ https://orcid.org/0000-0002-0734-5533 \\ Mee-Eun Kim \\ https://orcid.org/0000-0001-9332-532X
}

\section{REFERENCES}

1. IASP orofacial pain fact sheet. Burning mouth syndrome [Internet]. Washington, D.C.: International Association for the study of pain; c2016 [(updated 2016 Sep) cited 2019 Nov 15]. Available from: https://www.iasp-pain.org/Advocacy/GYAP2016Detail. aspx?ItemNumber=5573.

2. Feller L, Fourie J, Bouckaert M, Khammissa RAG, Ballyram R, Lemmer J. Burning mouth syndrome: aetiopathogenesis and principles of management. Pain Res Manag 2017;2017:1926269.

3. Souza FT, Santos TP, Bernardes VF, et al. The impact of burning mouth syndrome on health-related quality of life. Health Qual Life Outcomes 2011;9:57.

4. López-Jornet P, Camacho-Alonso F, Lucero-Berdugo M. Quality of life in patients with burning mouth syndrome. J Oral Pathol Med 2008;37:389-394.

5. Ni Riordain R, Moloney E, O'Sullivan K, McCreary C. Burning mouth syndrome and oral health-related quality of life: is there a change over time? Oral Dis 2010;16:643-647.

6. Tu TTH, Takenoshita M, Matsuoka H, et al. Current management strategies for the pain of elderly patients with burning mouth syndrome: a critical review. Biopsychosoc Med 2019;13:1.

7. Lee GS, Kim HK, Kim ME. Relevance of sleep, pain cognition, and psychological distress with regard to pain in patients with burning mouth syndrome. Cranio 2019. doi: 10.1080/08869634.2019.1681621. [Epub ahead of print]

8. Headache Classification Committee of the International Headache Society (IHS). The International Classification of Headache Disorders, 3rd edition (beta version). Cephalalgia 2013;33:629-808.

9. Cleeland CS, Ryan KM. Pain assessment: global use of the Brief 
Pain Inventory. Ann Acad Med Singapore 1994;23:129-138.

10. Yun YH, Mendoza TR, Heo DS, et al. Development of a cancer pain assessment tool in Korea: a validation study of a Korean version of the brief pain inventory. Oncology 2004;66:439-444.

11. Buysse DJ, Reynolds CF 3rd, Monk TH, Berman SR, Kupfer DJ. The Pittsburgh Sleep Quality Index: a new instrument for psychiatric practice and research. Psychiatry Res 1989;28:193-213.

12. Sullivan MJL, Bishop SR, Pivik J. The Pain Catastrophizing Scale: development and validation. Psychol Assess 1995;74:524-532.

13. Derogatis LR. SCL-90-R, administration, scoring \& procedures manual. Baltimore (MD): Clinical Psychology Research; 1977.

14. Adamo D, Pecoraro G, Fortuna G, et al. Assessment of oral health-related quality of life, measured by OHIP-14 and GOHAI, and psychological profiling in burning mouth syndrome: a casecontrol clinical study. J Oral Rehabil 2020;47:42-52.
15. Drage LA, Rogers RS 3rd. Burning mouth syndrome. Dermatol Clin 2003;21:135-145.

16. Adamo D, Schiavone V, Aria M, et al. Sleep disturbance in patients with burning mouth syndrome: a case-control study. J Orofac Pain 2013;27:304-313.

17. Benoliel R, Zini A, Zakuto A, et al. Subjective sleep quality in temporomandibular disorder patients and association with disease characteristics and oral health-related quality of life. J Oral Facial Pain Headache 2017;31:313-322.

18. Keilani M, Crevenna R, Dorner TE. Sleep quality in subjects suffering from chronic pain. Wien Klin Wochenschr 2018;130:31-36.

19. Adamo D, Sardella A, Varoni E, et al. The association between burning mouth syndrome and sleep disturbance: a case-control multicentre study. Oral Dis 2018;24:638-649. 